Gut, $1975,16,854-860$

\title{
Impaired anamnestic cellular immune response in patients with Crohn's disease
}

\author{
S. G. M. MEUWISSEN, P. Th. A. SCHELLEKENS, L. HUISMANS, AND \\ G. N. TYTGAT
}

From the Division of Gastroenterology, Willhelmina Gasthuis and Binnengasthuis, University of Amsterdam, and Laboratory for Experimental and Clinical Immunology, Central Laboratory of the Netherlands Red Cross Bloodtransfusion Service, Amsterdam, Holland

SUMMARY The cellular immune system was studied in patients with Crohn's disease (CD), not receiving corticosteroids, or azathioprine, by means of in vitro and in vivo methods. It was found, that the in vitro lymphocyte reactivity of $54 \mathrm{CD}$ patients after stimulation with a cocktail of antigens (varidase, trichophyton, candida, mumps, and PPD) was significantly depressed when compared with the response of 20 simultaneously cultured healthy controls $(P<0.001)$ or a group of 54 separately cultured healthy controls, matched for age and sex $(P<0.001)$. The lymphocyte response of a control group of 18 patients with malnutrition or malabsorption without any evidence of inflammatory bowel disease, was higher than the response of an equal number of CD cases, although the difference failed to reach significance. Intradermally injection of the same five antigens, as used in the antigen cocktail, showed a failure to react to any antigen in 13 out of $48 \mathrm{CD}$ patients, in comparison with three out of 48 matched healthy controls $(P<0.01)$. In both CD patients, as well as in healthy controls a significant correlation could be demonstrated between the number of positive skin tests, the area of skin induration, and the in vitro lymphocyte responsiveness after stimulation with the antigen cocktail. In the CD group no correlation was found between in vitro responsiveness and disease activity, as defined by a score of clinical and biochemical parameters. The depressed skin reactivity and the hyporesponsiveness in the lymphocyte transformation test after stimulation by an antigen cocktail suggest that depression of the anamnestic cellular immune response is a basic feature in patients with Crohn's disease.

Data regarding cellular immunity in Crohn's disease are not conclusive. Depression of delayed hypersensitivity was found by several authors (Blackburn et al., 1939; Phear, 1958; Williams, 1965; Jones et al., 1969). Others, however, reported normal skin reactivity (Binder, 1966; Fletcher and Hinton, 1967; Geffroy et al., 1971; Röpke, 1972; Bolton et al., 1974).

In vitro hyporesponsiveness in the lymphocyte transformation test has been documented (Parent $e t$ al., 1971; Guillou et al., 1973; Sachar et al., 1973; Richens et al., 1974), but many reports of unimpaired in vitro lymphocyte reactivity have also been published (Aas et al., 1972; Röpke, 1972; Asquith et al., 1973; Bird and Britton, 1974; Bolton et al., 1974).

To what extent the discrepancies that are mentioned are due to differences in technique is unsolved.

Received for publication 6 August 1975.
Furthermore, it is still largely unknown whether hyporesponsiveness in vivo or in vitro, if present in $\mathrm{CD}$, is in any way related to clinical activity of the disease, or caused by non-specific mechanisms, such as malabsorption, recent surgery, or immunosuppressive therapy (Asquith et al., 1973; Sachar et al., 1973).

Because of these conflicting data, we studied the cellular immune system in patients with $C D$ without immunosuppressive therapy and control subjects by testing both skin reactivity and in vitro lymphocyte transformation. In the in vitro tests, peripheral lymphocytes were not only stimulated with nonspecific plant mitogens, but also with an antigen cocktail, as described by Leguit et al., 1973. By this approach, we hoped to answer the following questions: Is the cellular immune system impaired in Crohn's disease? Is there a correlation between a diminished or absent skin reactivity and the in vitro 
response in the lymphocyte transformation test? Is the in vivo or in vitro reactivity in any way related to the clinical activity of the disease? Can hyporesponsiveness in Crohn's disease be due to nonspecific factors such as malabsorption or malnutrition?

\section{Methods}

\section{SUBJECTS}

Fifty-four patients with CD (mean age 34.8 years, range 17-60 years, 26 females and 28 males) were studied (group 1). Regional ileitis or ileocolitis was diagnosed in 39 cases, based on characteristic clinical, radiological, endoscopical and/or histological criteria. In 15 patients a diagnosis of $C D$ of the colon was made, according to criteria described elsewhere (to be published). Twenty-five patients had been operated upon for their CD and 17 of them showed evidence of recurrent disease at the time of the study. None of the patients received corticosteroids and/or azathioprine. Disease activity was arbitrarily scored as shown in Fig. 1: 31 patients were active; the non-active CD group, scoring less than 4 , consisted of six patients previously operated upon for their disase, but at present free from symptoms and without radiological evidence of recurrent disease. The other 25 patients in the nonactive group showed radiological or biochemical abnormalities, but had no or only mild complaints. The control group consisted of 20 healthy non- selected blood donors (group II) age 18-50 years, and 54 healthy subjects (group III) matched for age and sex. A further control group (group IV) consisted of 18 patients (mean age 49, range 28-70 years) with chronic pancreatitis with steatorrhoea or postgastrectomy diarrhoea, with no evidence of inflammatory bowel disease.

\section{Techniques}

\section{In vitro LYMPHOCYTE STUDIES}

Lymphocyte cultures were performed as described by Du Bois et al. (1973). Briefly the procedure was as follows: blood samples were defibrinated by shaking for 10 minutes with glass beads. Lymphocytes were separated from defibrinated blood by Ficoll-Isopaque gradient centrifugation. Lymphocyte suspensions were stored in liquid nitrogen (Eysvoogel and Van Loghem, 1969). After washing and cell counting the cells were resuspended in minimal essential medium (MEM-S, Gibco, New York, USA), buffered with Tris $(0.025 \mathrm{M})$, and supplemented with fresh heat-inactivated human $A B$ serum $(20 \%$ of the final volume), penicillin (100 IU per $\mathrm{ml})$, and streptomycin $(100 \mu \mathrm{g}$ per $\mathrm{ml})$. $0.2 \times 10^{6}$ lymphocytes in $1 \mathrm{ml}$ medium were cultured in polystyrene tubes with no stimulant added, or stimulated by a cocktail of antigens, containing varidase, trichophyton, candida, mumps, and PPD, the mixture containing each of the antigens so as to give an optimal final concentration (Leguit et al.,

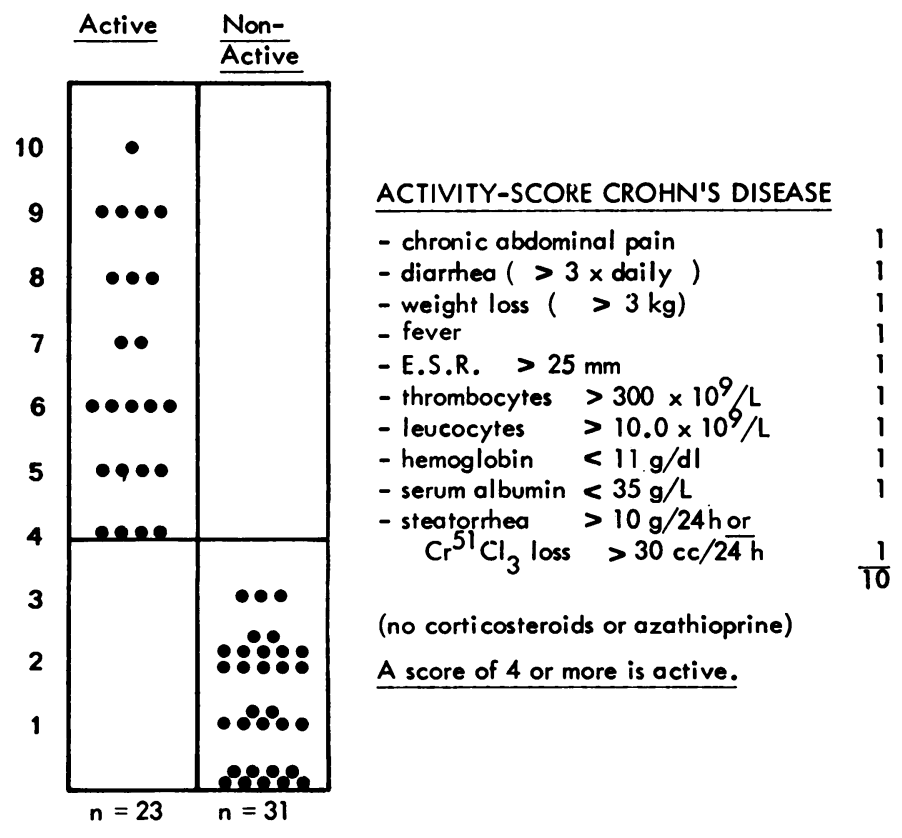

Fig. 1 Activity-score of 54 patients with Crohn's disease 
1973). For mitogen stimulation, poke-weed mitogen (PWM) was used at $25 \mu \mathrm{g} / \mathrm{ml}$ and phytohemagglutinin (PHA) at $50 \mu \mathrm{g} / \mathrm{ml}$; pilot studies showed no differences between $C D$ patients and controls when higher or lower PHA concentrations were used. Cultures were performed in triplicate. To determine DNA synthesis, ${ }^{2-14} \mathrm{C}$ thymidine $(0.075 \mu \mathrm{Ci}$, specific activity $6.25 \mathrm{mCi}$-mMol) was added 24 hours before harvesting. PHA stimulated cultures were harvested at day 3 , all other cultures at day 6 . Cells were collected and washed on glass fibre filters (Millipore, USA), which were then dried. Counting was performed for five minutes in a Packard liquid scintillation counter, using $2 \mathrm{ml}$ scintillation liquid. The means of triplicate outlines were expressed as crude counts per minute. No corrections were made for unstimulated lymphocyte activity, because statistical analysis showed no differences between crude or corrected counts.

Lymphocytes of group I and II were cultured simultaneously, while group III was studied separately, under comparable culture conditions. Group IV was cultured simultaneously with 17 non-selected CD patients from group I.

SKIN TESTING

Forty-eight of the $54 \mathrm{CD}$ patients (group I) and 48 age-and sex-matched healthy controls (group III) were skin tested with five different antigens $(0.1 \mathrm{ml}$ of a solution of varidase $50 \mathrm{USK} / \mathrm{SD} / \mathrm{ml}, 0.1 \mathrm{ml}$ tri- chophyton $0 \cdot 1 \%, 0 \cdot 1 . \mathrm{ml}$ candida $1: 300,0 \cdot 1 \mathrm{ml}$ mumps $20 \mathrm{CFU} / \mathrm{ml}$, and $0.1 \mathrm{ml}$ purified protein derivate $10 \mathrm{TU} / \mathrm{ml})$. The antigen solutions were injected intradermally on the volar surface of the forearm, after blood had been drawn for in vitro lymphocyte studies. Reactions were examined after 48 hours. An induration of $5 \mathrm{~mm}$ or more at two perpendicular diameters was considered positive.

\section{STATISTICAL ANALYSIS}

Results of in vitro tests on different days of cultures were analysed by a variant of Wilcoxon's sum of ranks test (two-sided), as described by Van Elteren (1960). Skin reactivity was analysed by the MantelHaenszel method (1959) and a correlation between the number of positive skin tests and the results in the antigen cocktail was studied by a variant of Kendall's rank correlation test (Terpstra, 1955). Spearman's rank correlation test was used to correlate skin induration and in vitro reactivity. For graphical purposes, all values were expressed on a log scale as crude count, the median of which was determined.

\section{Results}

\section{In vitro LYMPHOCYTE REACTIVITY}

The results of the lymphocyte culture experiments are summarized in Fig. 2. A significant depression in the unstimulated cultures was found in the CD

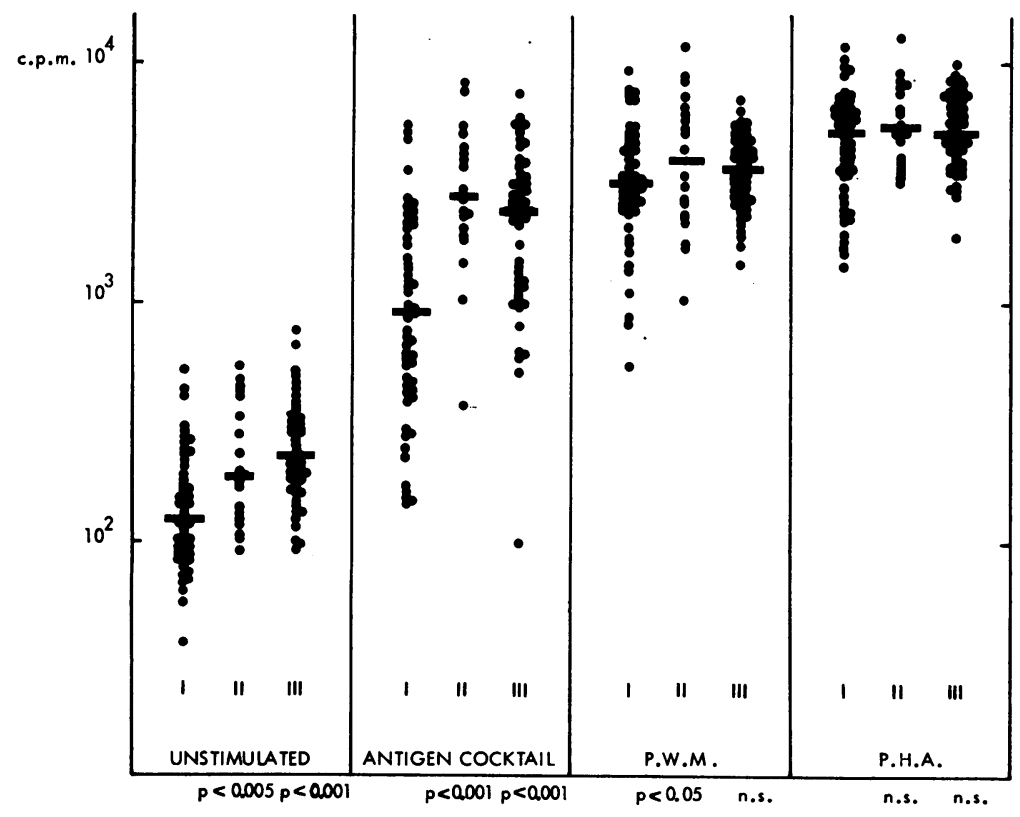

Fig. 2 In vitro lymphocyte responsiveness of 54 patients with Crohn's disease (group I), 20 simultaneously cultured healthy controls (group II), and 54 separately cultured healthy controls (group III). 
patients (group I), as compared with control groups II and III. In group I, the mean response to the antigen cocktail was significantly depressed, compared with group II $(\mathrm{P}<0.001)$ and III $(\mathrm{P}<0.001)$. There was also a difference of PWM responsiveness between group I and II (P $<0.05)$, although this is not immediately apparent from the Figure. No differences could be demonstrated in the reactivity to PHA. The in vitro responsiveness after stimulation with the antigen cocktail was not related to activity of the disease, sex, age, or localization of the disease, as shown in Fig. 3. However, there was a trend for patients with very low albumin levels (six cases) to show hyporesponsiveness only to the antigen cocktail. The responses of $17 \mathrm{CD}$ patients (group I) and 18 patients (group IV) are compared in Fig. 4. In the CD group, the reactivity after stimulation by the antigen cocktail, although substantially lower than the control response, failed to reach significance $(P<0 \cdot 10)$. Differences in reactivity to PWM or PHA were not observed.

\section{SKIN REACTIVITY}

The results of the skin tests are shown in Fig. 5. Thirteen CD patients, 10 females and three males, of whom an equal number were active and nonactive, failed to develop a positive skin test, in contrast with three control subjects (group III); this difference was significant $(P<0.01)$. No difference was found between the mean overall number of positive skin reactions in the CD group (1.6) and in the controls $(2 \cdot 0)$. No differences in reactivity to the individual antigens was found, except for a significantly higher number of positive reactions to candida-antigen in the control group ( $P<0.01)$.

Figure 6 shows that a correlation exists between the number of positive skin tests and the lymphocyte reactivity after stimulation with the antigen cocktail, both in the CD group and controls. The total area of skin induration was also positively correlated with the in vitro reactivity in both CD patients $(r=0.401$, $P<0.01)$ and normal subjects $(r=0.306, \mathrm{P}<0.05)$.

\section{Discussion}

We have shown in this study that patients with Crohn's disease manifest a depressed cellular immune response, compared with healthy controls. This is revealed by a decrease in both skin reactivity and in the lymphocyte transformation test after stimulation with a combination of bacterial, fungal, and viral antigens; the results of both methods were positively correlated. We were unable to demonstrate differences in reactivity to PHA or PWM between patients with $C D$, healthy controls, or patients with malnutrition or malabsorption, not due to inflammatory bowel disease. Therefore, lymphocyte stimulation by an antigen cocktail may be useful in testing the anamnestic cellular responsiveness in CD. The value of this antigen cocktail in this regard was also shown by Leguit et al. (1973) in patients with thermal injury and by Schellekens et al. (1974), in patients during immunosuppressive therapy. In contrast with the lymphocytic response

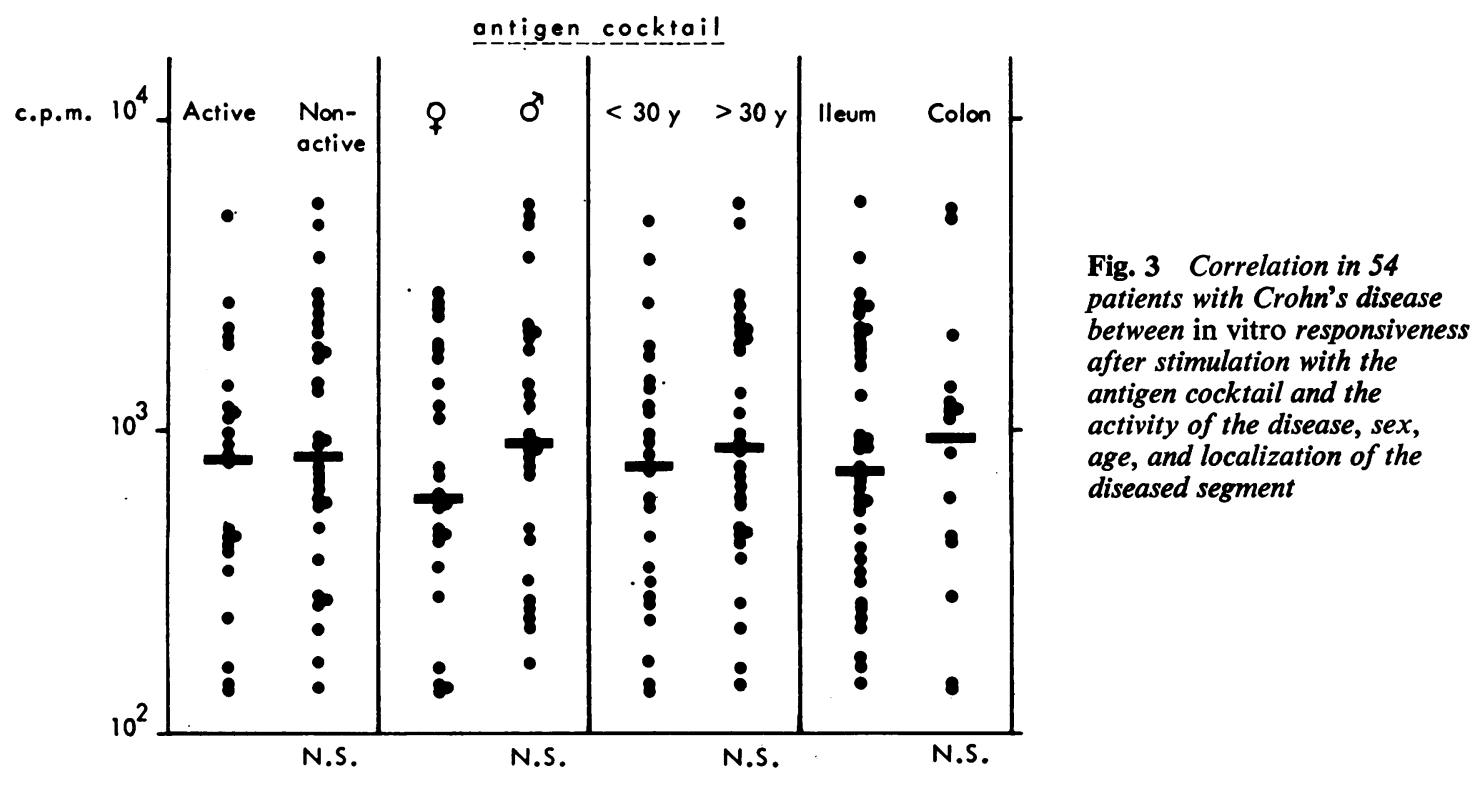




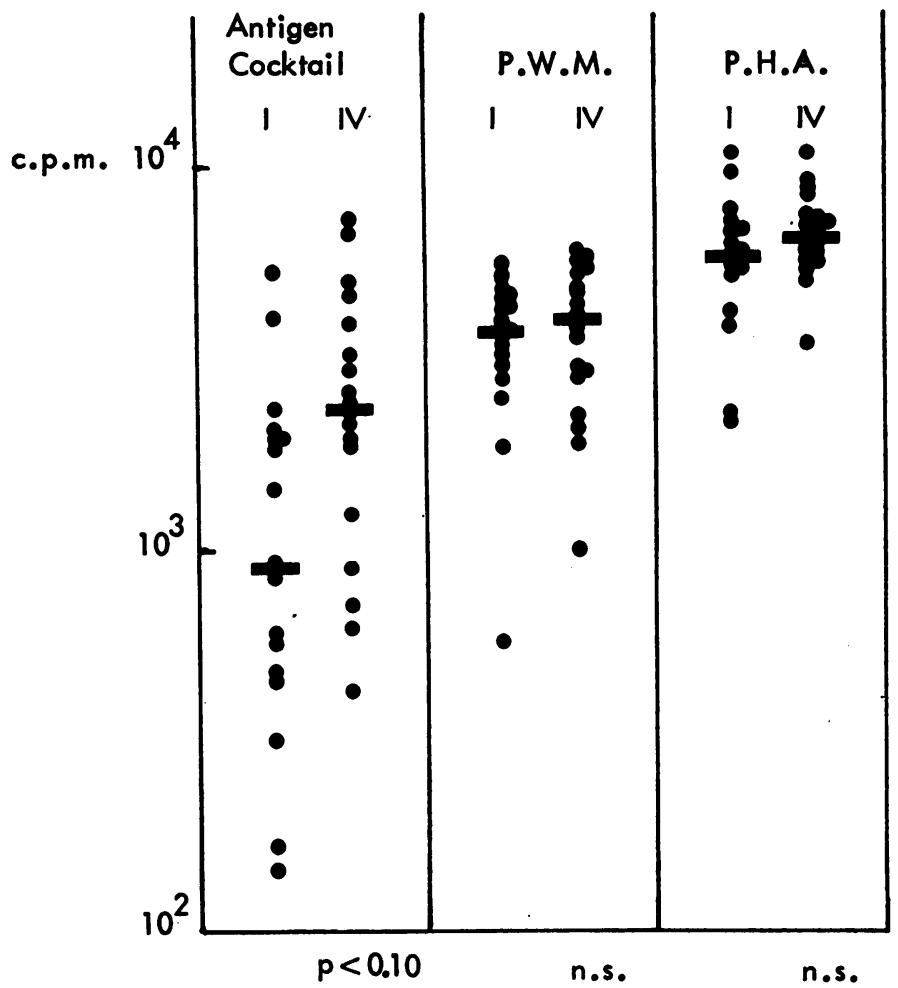

Fig. 4 Comparison of in vitro responsiveness between 17 patients with Crohn's disease (group I) and 18 patients with malabsorption, without evidence of inflammatory bowel disease (group IV)

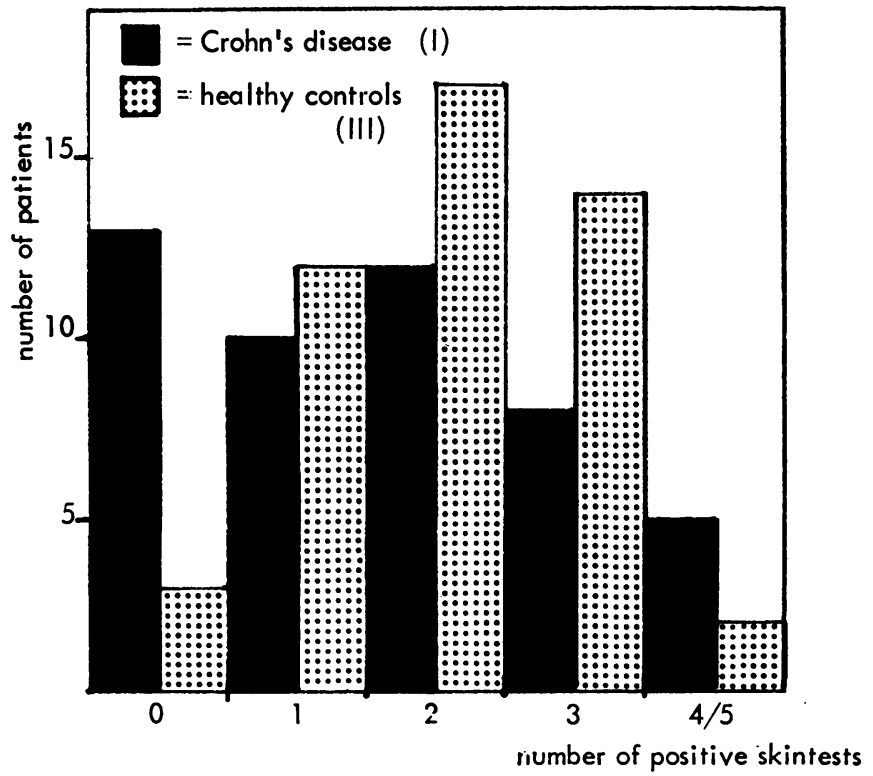

Fig. 5 Diagram of number of positive skin tests in 48 patients with Crohn's disease and 48 healthy controls (group III). 


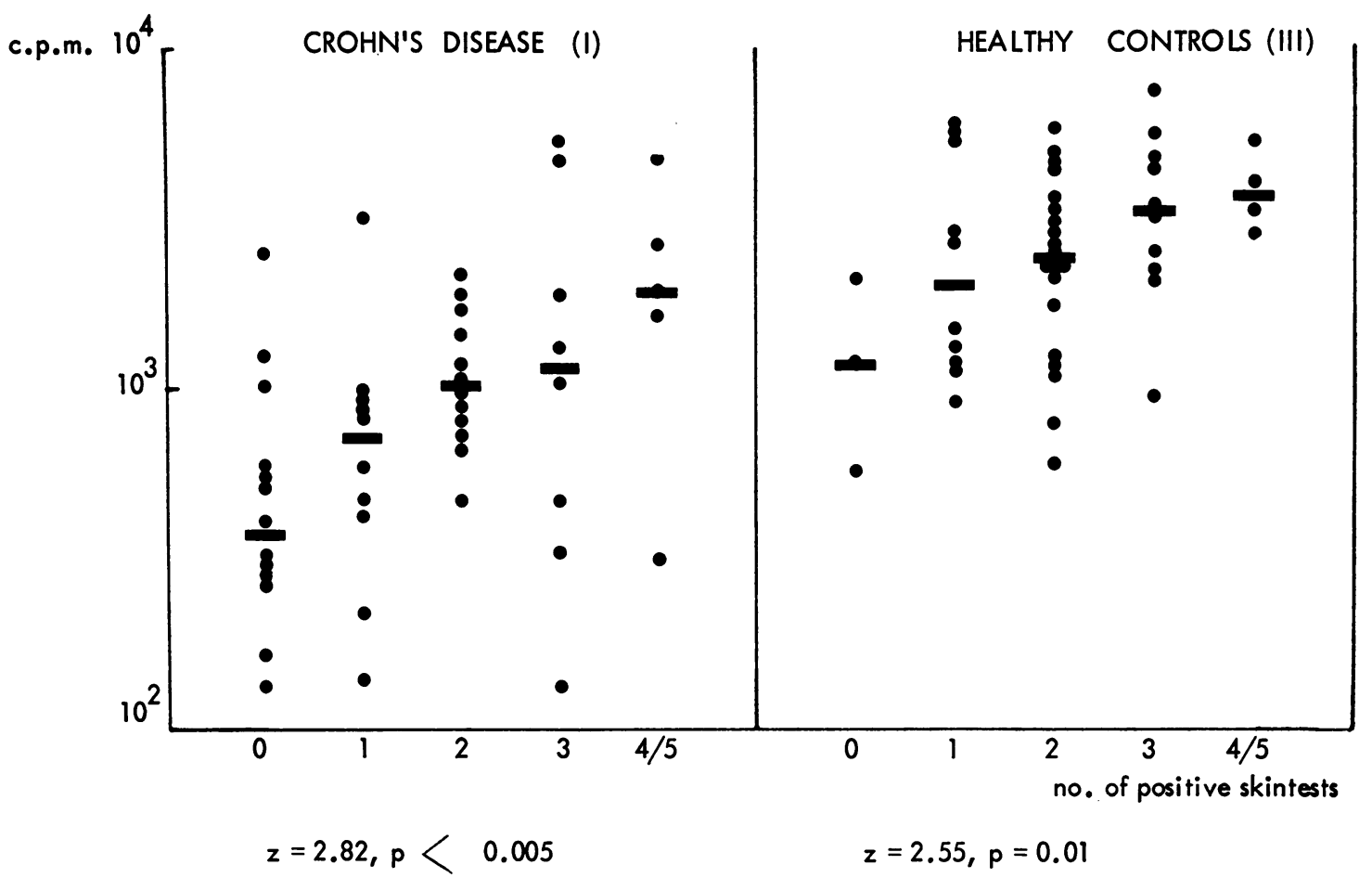

Fig. 6 Correlation between the in vitro responsiveness after stimulation with the antigen cocktail and the number of positive skin tests in Crohn's disease and healthy controls (group III)

of CD patients in the study of Aas et al. (1972) and Asquith et al. (1973), our CD patients showed also a diminished response in the unstimulated cultures. It is highly speculative, at present, to assume that the basal lymphocytic hyporesponsiveness might also explain the lowered response to the antigen cocktail, because under identical culture conditions no differences were found when stimulating with PWM or PHA.

It might be that hyporesponsiveness in CD is due to non-specific factors such as malnutrition or malabsorption, as postulated by Bird and Britton (1974). However, malnutrition was not a frequent finding in our CD patients. Most of the non-active cases showed a normal haemoglobin, serum albumin, cholesterol, vitamin B12, and folic acid levels, but still showed a decreased response to the antigen cocktail. We can also rule out in this study other non-specific factors such as recent surgery or immunosuppressive therapy. Although the majority of our subjects received azulfidine, the metabolite of which, 5-aminosalicylate, might suppress lymphocyte blastogenesis, recent studies failed to demonstrate such effect in CD patients (Parent et al., 1971;
Sachar et al., 1973). Further arguments in favour of an intrinsic hyporesponsive cellular immunity in $\mathrm{CD}$ are the absence of correlation between lymphocyte blastogenesis and disease activity in patients with $C D$, and the substantially higher response of patients with non-inflammatory malnutrition or malabsorption, the latter being almost identical with the value obtained in healthy controls.

In conclusion, our results suggest an impaired cellular immune response in patients with $\mathrm{CD}$. It should be emphasized, however, that this hyporesponsiveness is confined to the anamnestic response to antigens. Jones et al. (1969) reported that also primary immune responses might be depressed in $\mathrm{CD}$. We have been able to confirm this latter finding by testing delayed skin reactivity to dinitrochlorobenzene in $35 \mathrm{CD}$ patients (unpublished data). However, further studies are needed, to analyse whether this hyporesponsiveness is due to changes at the cellular level or merely reflects a redistribution of the various subclasses of peripheral lymphocytes, or perhaps a shift of responsive cells towards the diseased gut, specifically committed to a so-far unknown bacterial, viral, or fungal antigen. 


\section{References}

Aas, J., Huizenga, K. A., Newcomer, A. D., and Shorter, R. G. (1972). Inflammatory bowel disease: lymphocytic responses to nonspecific stimulation in vitro. Scandinavian Journal of Gastroenterology, 7, 299-303.

Asquith, P., Kraft, S. C., and Rothberg, R. M. (1973). Lymphocyte responses to nonspecific mitogens in inflammatory bowel disease. Gastroenterology, 65, 1-7.

Binder, H. J., Spiro, H. M., and Thayer, W. R. (1966). Delayed hypersensitivity in regional enteritis and ulcerative colitis. American Journal of Digestive Diseases, 11, 572-574.

Bird, A. G., and Britton, S. (1974). No evidence for decreased lymphocyte reactivity in Crohn's disease. Gastroenterology, 67, 926-932.

Blackburn, G., Hadfield, G., and Hunt, A. H. (1939). Regional ileitis. St. Bartholomew's Hospital Report, 72, 181-224.

Bolton, P. M., James, S. L., Newcombe, R. G., Whitehead, R. H., and Hughes, L. E. (1974). The immune competence of patients with inflammatory bowel disease. Gut, 15, 213-219.

Du Bois, M. J. G. J., Huismans, D. R., Schellekens, P. Th. A., and Eijsvoogel, V. P. (1973). Investigation and standardization of the conditions for micro-lymphocyte cultures. Tissue Antigens, 3, 402-409.

Elteren, Van P. H. (1960). On the combination of independent two samples tests of Wilcoxon. Institute Internationale de Statistique, Bulletin, 37, 351-360.

Eysvoogel, V. P., and Van Loghem, J. J. (1969). Preservation of lymphocytes at cryogenic temperatures. In Manual of Tissue Typing Techniques, p. 62. National Institute of Allergy and Infectious Diseases, Transplantation Immunology Branch: Bethesda, Md.

Fletcher, J., and Hinton, J. M. (1967). Tuberculin sensitivity in Crohn's disease. Lancet, 2, 753-754.

Geffroy, Y., Colin, R., Hecketsweiler, P. H., and Segrestin, M. (1971). Traitement de la maladie de Crohn par le B.C.G. Archives des Maladies de l'Appareil Digestif, 64, 299-308.
Guillou, P. J., Brennan, T. G., and Giles, G. R. (1973). Lymphocyte transformation in the mesenteric lymph nodes of patients with Crohn's disease. Gut, 14, 20-24.

Jones, J. V., Housley, J., Ashurst, P. M., and Hawkins, C. F. (1969). Development of delayed hypersensitivity to dinitrochlorobenzene in patients with Crohn's disease. Gut, 10, 52-56.

Leguit, P., Meinesz, A., Huismans, L., and Eijsvoogel, V. P. (1973). The use of an antigen cocktail in the lymphocyte transformation test. Clinical and Experimental Immunology, 14, 149-152.

Mantel, N., and Haenszel, W. (1959). Statistical aspects of the analysis of data from retrospective studies of disease. Journal of the National Cancer Institute, 22, 719-748.

Parent, K., Barrett, J., and Wilson, I. D. (1971). Investigation of the pathogenic mechanisms in regional enteritis with in vitro lymphocyte cultures. Gastroenterology, 61, 431-439.

Phear, D. N. (1958). The relation between regional ileitis and sarcoidosis. Lancet, 2, 1250-1251.

Richens, E. R., Williams, M. J., Gough, K. R., and Ancill, R. J. (1974). Mixed-lymphocyte reaction as a measure of immunological competence of lymphocytes from patients with Crohn's disease. Gut, 15, 24-28.

Röpke, C. (1972). Lymphocyte transformation and delayed hypersensitivity in Crohn's disease. Scandinavian Journal of Gastroenterology, 7, 671-677.

Sachar, D. B., Taub, R. N., Brown, S. M., Present, D. H., Korelitz, B. I., and Janowitz, H. D. (1973). Impaired lymphocyte responsiveness in inflammatory bowel disease. Gastroenterology, 64, 203-209.

Schellekens, P. Th. A., v.d. Borne, A. E. G. Kr., Wilmink, J. M., De Vet, B. J. C. M., and Eijsvoogel, V. P. (1974). Lymphocyte reactivity in vitro and clinical immunosuppression. Tissue Antigens, 4, 407-408. (Abstract).

Terpstra, T. J. A. (1955-1956). A generalization of Kendall's rank correlation statistic. Proceedings Koninklijke Nederlande Akademie van Wetenschappen, Series A, 58-59.

Williams, W. J. (1965). A study of Crohn's syndrome using tissue extracts and the Kveim and Mantoux tests. Gut, 6, 503-505. 